\title{
SOME NECESSARY CONDITIONS FOR RADIAL FOURIER MULTIPLIERS
}

\author{
WALTER TREBELS
}

\begin{abstract}
Necessary conditions in terms of differentiability and growth properties for a radial function $m(|z|)$ to be a Fourier multiplier of type $(p, q)$ are given and compared with sufficient ones.
\end{abstract}

Introduction. The starting point of the following is to be seen in the observation (see [7, p. 114]) that a radial multiplier of type $(p, p), p$ $<2 n /(n+1)$, has to be continuous everywhere, except possibly the origin, and the following strengthening of this result due to Tomas [9].

THEOREM A. If $f$ is a radial function on $R^{n}$ and $f$ is in $L^{p}\left(R^{n}\right)$ for $1 \leqslant p<2 n /(n+1+2 \kappa), \kappa=k+\delta^{\prime}, k$ integer and $0 \leqslant \delta^{\prime}<1$, then $f^{\prime}(|z|)$ $=f^{\wedge}(t), z \in R^{n}, t>0$, has $k$ continuous derivatives away from the origin and

$$
\left|f^{\wedge(k)}(t+s)-f^{\wedge(k)}(t)\right| \leqslant C\left(t_{0}\right) s^{\delta^{\prime}} \text { for all } t \geqslant t_{0}>0 .
$$

Schoenberg [5] has proved that the Hankel transform (which arises as a generalization of the Fourier transform of a radial integrable function on Euclidean $n$-space) has $[(n-1) / 2]$ derivatives; in [6] Schwartz has improved Schoenberg's result, actually obtaining a stronger version of Theorem A in case $p=1$. Our main result, Theorem 1 , represents a combination of the results of Schwartz and Tomas in case of ordinary derivatives; the extension to fractional ones is new. Furthermore, Theorem 1 allows us to deduce necessary conditions for radial Fourier $M_{p}^{q}$ multipliers (in an elementary way). A comparison of these conditions with sufficient ones enlightens to some extent the structure of radial Fourier multipliers.

The author is obliged to the referee for pointing out the papers of Schoenberg [5] and Schwartz [6].

The following notation will be used: $y, z \in R^{n}, s, \ldots, x \in R ; S$ is the set of all infinitely differentiable functions on $R^{n}$, rapidly decreasing at infinity. Let $L^{p}\left(R^{n}\right), n \geqslant 2$, be the standard Lebesgue space of $p$ th power integrable functions with norm

$$
\|f\|_{p}=\left(\int_{R^{n}}|f(y)|^{p} d y\right)^{1 / p}
$$

Received by the editors September 9, 1975.

AMS (MOS) subject classifications (1970). Primary 42A18.

Key words and phrases. Radial Fourier multipliers, fractional differentiation.

(c) American Mathematical Society 1976 
and $L_{\mathrm{rad}}^{p}$ be the subset of $L^{p}$ consisting of radial functions. The Fourier transform of $f \in L_{\mathrm{rad}}^{p}, 1 \leqslant p<2 n /(n+1)$, is defined by [8, p. 155]

$$
f^{\wedge}(|z|)=f^{\wedge}(t)=\int_{0}^{\infty} K_{(n-2) / 2}(t s) f(s) s^{n-1} d s \quad(n \geqslant 2),
$$

where $K_{a}(s)=s^{-a} J_{a}(s), J_{a}(s)$ denoting the Bessel function of order $a$, and where we have set in abuse of notation $f^{\wedge}(|z|)=f^{\wedge}(t), f(|y|)=f(s)$. By Hölder's inequality $\left(1 / p+1 / p^{\prime}=1\right), f^{\wedge}(|z|)$ exists for $z \neq 0$, since with the aid of Lemma 3.11 in [8, p. 158]

$$
\begin{aligned}
\sup _{t>0}\left|t^{n / p^{\prime}} f^{\wedge}(t)\right| \leqslant & \left(\int_{0}^{\infty}|f(s)|^{p} s^{n-1} d s\right)^{1 / p} \\
& \cdot\left(\int_{0}^{\infty}\left|K_{(n-2) / 2}(s)\right|^{p^{\prime}} s^{n-1} d s\right)^{1 / p^{\prime}} \leqslant C_{p}\|f\|_{p}
\end{aligned}
$$

in the prescribed $p$-range.

To state our main result we need the definition of a fractional derivative in the sense of J. Cossar (see [10, p. 31]): Consider for $0<\delta<1$ the fractional integral operator

$$
I_{w}^{1-\delta}[g](t)=\frac{1}{\Gamma(1-\delta)} \int_{t}^{w}(s-t)^{-\delta} g(s) d s
$$

and define

$$
g^{(\delta)}(t)=-\lim _{w \rightarrow \infty}(d / d t) I_{w}^{1-\delta}[g](t) .
$$

Usual differentiation of $g^{(\delta)}$ yields pure fractional derivatives of order $\kappa$ $=k+\delta$, i.e.,

$$
g^{(\kappa)}(t)=(d / d t)^{k} g^{(\delta)}(t) .
$$

If $\kappa$ is an integer, take ordinary derivatives; hence $g^{(\kappa)}$ is explained for all $\kappa \geqslant 0$. Our main result now reads:

Theorem 1. If $f \in L_{\mathrm{rad}}^{p}, 1 \leqslant p<2 n /(n+1)$, then

$$
\sup _{t>0}\left|t^{\kappa+n / p^{\prime}} f^{\mathcal{(}(\kappa)}(t)\right| \leqslant C_{p}\|f\|_{p}
$$

provided $0 \leqslant \kappa<n(1 / p-1 / 2)-1 / 2$.

Proof. First let $\kappa=k$ be an integer; then one may differentiate (1) $k$ times under the integral (see [9]). Observing that $K_{a}^{\prime}(s)=-s K_{a+1}(s)$ (see [8, p. 154]), $t^{n / p^{\prime}}(t(d / d t))^{i} f^{\wedge}(t), i=1, \ldots, k$, can be estimated by a linear combination of the terms $(j=1, \ldots, i)$

$$
\begin{aligned}
& \left|t^{n / p^{\prime}} t^{2 j} \int_{0}^{\infty} K_{(n-2) / 2+j}(s t) s^{2 j} f(s) s^{n-1} d s\right| \\
& \leqslant C\|f\|_{p}\left(\int_{0}^{\infty}\left|s^{2 j} K_{(n-2) / 2+j}(s)\right|^{p^{\prime}} s^{n-1} d s\right)^{1 / p^{\prime}} \\
& \leqslant C_{p}^{\prime}\|f\|_{p}
\end{aligned}
$$


the latter being true by Hölder's inequality. Hence,

$$
\sup _{t>0}\left|t^{n / p^{\prime}}\left(t \frac{d}{d t}\right)^{j} f^{\wedge}(t)\right| \leqslant C_{p}^{\prime \prime}\|f\|_{p}, \quad j=1, \ldots, k
$$

and the assertion follows by observing that

$$
t^{k} f^{\wedge(k)}(t)=\sum_{j=1}^{k} C_{j, k}\left(t \frac{d}{d t}\right)^{j} f^{\wedge}(t)
$$

Next, let $0<\kappa=\delta<1$. First we note that we can equivalently define

$$
g^{(\delta)}(t)=-\lim _{w \rightarrow \infty}(d / d t) I_{w+t}^{1-\delta}[g](t)
$$

if $g(t)$ is bounded for large $t$. Now we show that $f^{-(\delta)}(t)$ exists for each $t \geqslant t_{0}>0$. To this end, fix $t$ and choose $w$ sufficiently large. First observe that

$$
\begin{aligned}
f^{\wedge}(s)= & \int_{0}^{\infty} K_{(n-2) / 2}(s x) f(x) x^{n-1} d x \\
= & \int_{0}^{\infty} K_{(n-2) / 2}(x(s+w-t)) f(x) x^{n-1} d x \\
& -\frac{d}{d s} \int_{t}^{w} \int_{0}^{\infty}\left\{K_{(n-2) / 2}(x(u+s-t))-K_{(n-2) / 2}(x u)\right\} f(x) x^{n-1} d x d u
\end{aligned}
$$

and that

$$
\frac{d}{d t} \int_{t}^{w+t}(s-t)^{-\delta} \int_{0}^{\infty} K_{(n-2) / 2}(x(s+w-t)) f(x) x^{n-1} d x d s \equiv 0 .
$$

Then integrate by parts to obtain

$$
\begin{aligned}
f^{\wedge}(\delta)(t)= & -\lim _{w \rightarrow \infty} \frac{d}{d t} \frac{1}{\Gamma(1-\delta)} \int_{t}^{w+t}(s-t)^{-\delta} f^{\wedge}(s) d s \\
= & \lim _{w \rightarrow \infty} \frac{d}{d t} \frac{(s-t)^{-\delta}}{\Gamma(1-\delta)} \int_{t}^{w} \int_{0}^{\infty}\left\{K_{(n-2) / 2}(x(u+s-t))\right. \\
& \left.\quad-K_{(n-2) / 2}(x u)\right\}\left.f(x) x^{n-1} d x d u\right|_{s=t} ^{w+t} \\
& -\lim _{w \rightarrow \infty} \frac{d}{d t} \frac{1}{\Gamma(-\delta)} \int_{t}^{w+t}(s-t)^{-\delta-1} \int_{t}^{w} \int_{0}^{\infty}\{\cdots\} f(x) x^{n-1} d x d u d s .
\end{aligned}
$$

The first term on the right side at $s=t+w$ (inside the limit-sign) behaves like $O\left(w^{-\delta}\right)$ for large $w$ and, therefore, vanishes in the limit; at $s=t$ it vanishes identically if one uses Theorem A with $\delta<\delta^{\prime}$. Furthermore, again by Theorem A and relation (2), the triple integral converges absolutely for $0<t_{0} \leqslant t<w, w$ fixed; thus, by Fubini's theorem and a change of the $s$ variable,

$$
\begin{aligned}
f^{\sim(\delta)}(t)=-\lim _{w \rightarrow \infty} \frac{d}{d t} \int_{t}^{w} \frac{1}{\Gamma(-\delta)} \int_{0}^{w} s^{-\delta-1} \\
\cdot \int_{0}^{\infty}\left\{K_{(n-2) / 2}(x(u+s))-K_{(n-2) / 2}(x u)\right\} f(x) x^{n-1} d x d s d u \\
=\frac{-1}{\Gamma(-\delta)} \int_{0}^{\infty} s^{-\delta-1} \int_{0}^{\infty}\left\{K_{(n-2) / 2}(x(t+s))-K_{(n-2) / 2}(x t)\right\} f(x) x^{n-1} d x d s,
\end{aligned}
$$


the double integral being absolutely convergent by Theorem A and (2) for $t \geqslant t_{0}>0$. Thus it follows by Hölder's inequality that

$$
\begin{aligned}
& \left|t^{\delta+n / p^{\prime}} f^{\sim(\delta)}(t)\right| \leqslant C\|f\|_{p} \int_{0}^{\infty} s^{-\delta-1}\left(\int_{0}^{\infty}\left|t^{\delta}\{\cdots\}\right|^{p^{\prime}} t^{n} x^{n} \frac{d x}{x}\right)^{1 / p^{\prime}} d s \\
& \quad=C\|f\|_{p} \int_{0}^{\infty} s^{-\delta-1}\left(\int_{0}^{\infty}\left|K_{(n-2) / 2}(u(1+s))-K_{(n-2) / 2}(u)\right|^{p^{\prime}} u^{n-1} d u\right)^{1 / p^{\prime}} d s .
\end{aligned}
$$

Clearly, $\int_{1}^{\infty}\left(\int_{0}^{\infty}\right)$ converges absolutely by $(2)$; thus consider $\int_{0}^{1}\left(\int_{0}^{\infty}\right)$, split the inner integration into three integrations over $(0,1),(1,1 / s),(1 / s, \infty)$, use Minkowski's inequality, and denote the resulting double integrals by $I_{3}, I_{4}, I_{5}$, respectively. $I_{5}$ is easily estimated without using the difference; $I_{3}$ and $I_{4}$ are estimated analogous to Tomas [9]:

$$
\begin{aligned}
\mathrm{I}_{3} & =\int_{0}^{1} s^{-\delta-1}\left(\int_{0}^{1}\left|K_{(n-2) / 2}(u(1+s))-K_{(n-2) / 2}(u)\right|^{p^{\prime}} u^{n-1} d u\right)^{1 / p^{\prime}} d s \\
& \leqslant \int_{0}^{1} s^{-\delta}\left(\int_{0}^{1} u^{n-1} d u\right)^{1 / p^{\prime}} d s,
\end{aligned}
$$

where we used the mean value theorem

$$
\left|K_{(n-2) / 2}(u(1+s))-K_{(n-2) / 2}(u)\right| \leqslant u s \sup _{u \leqslant x \leqslant u+u s}\left|x K_{n / 2}(x)\right|
$$

and the standard properties of the Bessel function (see [8, Chapter IV]). Analogously, choosing $\delta^{\prime}, 0<\delta<\delta^{\prime}<n(1 / p-1 / 2)-1 / 2$, there holds

$$
\mathrm{I}_{4} \leqslant \int_{0}^{1} s^{-1-\delta} s^{\delta^{\prime}}\left(\int_{1}^{1 / u}\left|u^{\delta^{\prime}} u^{1-(n+1) / 2}\right|^{p^{\prime}} u^{n-1} d u\right)^{1 / p^{\prime}} d s<\infty .
$$

Hence the assertion is true for all $\delta, 0<\delta<n(1 / p-1 / 2)-1 / 2 \leqslant 1$. The general case $\kappa>1$ now follows by a combination of both methods, observing that

$$
\begin{array}{r}
f^{(\kappa)}(t)=C \int_{0}^{\infty} s^{-\delta-1} \int_{0}^{\infty}\left\{\left(\frac{d}{d t}\right)^{k} K_{(n-2) / 2}(x(t+s))-\left(\frac{d}{d t}\right)^{k} K_{(n-2) / 2}(x t)\right\} \\
\cdot f(x) x^{n-1} d x d s .
\end{array}
$$

Corollary. Let $m(|z|) \in M_{p}^{p}, 1<p<2 n /(n+1)$, then

$$
\sup _{t>0}\left|t^{\kappa} m^{(\kappa)}(t)\right| \leqslant C\|m\|_{M p}
$$

provided $0 \leqslant \kappa<n(1 / p-1 / 2)-1 / 2$.

Proof. First let $\kappa=k$ be an integer; choose $g_{0} \in S$ such that

$$
g_{0}(t)=g_{0}(|z|)= \begin{cases}1, & 2^{-1} \leqslant|z| \leqslant 2 \\ 0, & |z| \leqslant 2^{-2},|z| \geqslant 2^{2}\end{cases}
$$


and set $g_{i}(t)=g_{0}\left(2^{-j} t\right), j=0, \pm 1, \ldots$ Then, $\mathscr{F}^{-1}$ denoting the inverse Fourier transformation, $\left\|\mathscr{F}^{-1}\left[g_{j}(|z|)\right]\right\|_{p}=2^{j n / p^{\prime}}\left\|\mathscr{F}^{-1}\left[g_{0}\right]\right\|_{p} \leqslant C 2^{j n / p^{\prime}}$. Now $m(|z|) \in M_{p}^{p}$ implies $m(|z|) g_{j}(|z|) \in\left[L_{\mathrm{rad}}^{p}\right]^{\wedge}$ and, therefore, by Theorem 1

$$
\begin{aligned}
\sup _{t>0}\left|t^{k+n / p^{\prime}}\left(\frac{d}{d t}\right)^{k}\left[m(t) g_{j}(t)\right]\right| & \leqslant C\left\|\mathscr{F}^{-1}[m] * \mathscr{F}^{-1}\left[g_{j}\right]\right\|_{p} \\
& \leqslant C\|m\|_{M p}\left\|\mathscr{F}^{-1}\left[g_{j}\right]\right\|_{p} \leqslant C^{\prime} 2^{j n / p^{\prime}}\|m\|_{M p} .
\end{aligned}
$$

But this implies

$$
\sup _{2^{j-1}<t<2^{j+1}}\left|t^{k} m^{(k)}(t)\right| \leqslant C\|m\|_{M_{p} p}
$$

uniformly in $j$, and hence the assertion. If $\kappa$ is not an integer, choose a radial partition of unity with $e_{j}(t)=e_{0}\left(2^{-j} t\right), e_{0}(|z|) \in S$ such that

$$
e_{0}(t)=\left\{\begin{array}{ll}
1, & 2^{-1 / 3} \leqslant t \leqslant 2^{1 / 3}, \\
0, & t \leqslant 2^{-2 / 3}, t \geqslant 2^{2 / 3},
\end{array} \quad \sum_{j=-\infty}^{\infty} e_{j}(t)=1, t>0 .\right.
$$

Then, as before,

$$
\begin{aligned}
& \sup _{2^{j-1} \leqslant t \leqslant 2^{j+1}}\left|t^{\kappa+n / p^{\prime}} m^{(\kappa)}(t)\right| \leqslant \sum_{i=j-1}^{\infty} \sup _{2^{j-1} \leqslant t \leqslant 2^{j+1}}\left|t^{\kappa+n / p^{\prime}}\left(\frac{d}{d t}\right)^{\kappa}\left[m(t) e_{i}(t)\right]\right| \\
& \leqslant C\|m\|_{M^{\prime}} 2^{j n / p^{\prime}}+\left(2^{j+1}\right)^{\kappa+n / p^{\prime}} \sum_{i=j+2}^{\infty} \sup _{2^{j-1} \leqslant t \leqslant 2^{j+1}} \mid\left(\frac{d}{d t}\right)^{\kappa}\left[m(t) e_{i}(t)\right] .
\end{aligned}
$$

Now observe that for $t \in\left[2^{j-1}, 2^{j+1}\right]$ one has

$$
\left(\frac{d}{d t}\right)^{\kappa}\left[m(t) e_{i}(t)\right]=C \int_{2^{i-2 / 3}}^{2^{i+2 / 3}}(s-t)^{-\kappa-1} m(s) e_{j}(s) d s,
$$

which implies for $i \geqslant j+2$

$$
\sup _{2^{j-1} \leqslant t \leqslant 2^{j+1}}\left|\left(\frac{d}{d t}\right)^{\kappa}\left[m(t) e_{i}(t)\right]\right| \leqslant C\left(2^{i-1}\right)^{-\kappa}\|m\|_{\infty}
$$

and, therefore,

$$
\begin{aligned}
\sup _{2^{j-1} \leqslant t \leqslant 2^{j+1}}\left|t^{\kappa}\left(\frac{d}{d t}\right)^{\kappa} m(t)\right| & \leqslant C^{\prime}\|m\|_{M_{p} p}+C^{\prime}\left(2^{j+1}\right)^{\kappa}\|m\|_{M_{p} p} \sum_{i=j+2}^{\infty}\left(2^{i-1}\right)^{-\kappa}
\end{aligned}
$$

which is uniformly bounded in $j$. Hence the assertion follows.

REMARK. For a quasi-characterization of radial Fourier multipliers it is convenient to introduce the following classes: that

(i) $W B V_{\kappa+1}^{a}, \kappa \geqslant 0, a \geqslant 0$, consists of all sufficiently smooth functions such

$$
\|e\|_{W B V_{\kappa+1}^{a}}:=\left\|t^{a} e(t)\right\|_{\infty}+\sup _{j} \int_{2^{j}}^{2^{j+1}} t^{\kappa+a}\left|d e^{(\kappa)}(t)\right|<\infty .
$$


(ii) $W B V_{\beta, q}^{a}, 1<q<\infty, \beta \geqslant 1 / q, a \geqslant 0$ consists of all sufficiently smooth functions such that

$$
\|e\|_{W B V_{\beta, q}^{a}}:=\left\|t^{a} e(t)\right\|_{\infty}+\left(\sup _{j} \int_{2^{j}}^{2^{j+1}}\left|t^{\beta+a} e^{(\beta)}(t)\right|^{q} \frac{d t}{t}\right)^{1 / q}<\infty
$$

(this is a generalization of (i) inspired by the localized Bessel potential spaces in Connett and Schwartz [3]).

(iii) $W B V_{\beta, \infty}^{a}, \beta>0, a \geqslant 0$ consists of all sufficiently smooth functions such that

$$
\|e\|_{W B V_{\beta, \infty}^{a}}:=\left\|t^{a} e(t)\right\|_{\infty}+\left\|t^{\beta+a} e^{(\beta)}(t)\right\|_{\infty}<\infty .
$$

Now combining the above Corollary with results of Bonami and Clerc [1] we obtain

$$
W B V_{\beta+1}^{0} \subset M_{p}^{p}(\mathrm{rad}) \subset W B V_{\kappa, \infty}^{0}, \quad 1<p<2 n /(n+1),
$$

where $\kappa<n(1 / p-1 / 2)-1 / 2$ and $\beta$ integer, $\beta>(n-1)(1 / p-1 / 2)$ (in a forthcoming paper it will be shown by using results of [3] and [11] that this holds also for fractional $\beta$ ). For $p$ near 1, i.e., $\beta \approx \kappa, W B V_{\beta+1}^{0}$ and $W B V_{\kappa, \infty}^{0}$ are not so far away from each other, since $W B V_{\kappa+1}^{0} \subset W B V_{\kappa, \infty}^{0}$, but $W B V_{\kappa+1}^{0}$ $\llbracket W B V_{\kappa+\varepsilon, \infty}^{0}$ for any $\varepsilon>0$, as the example $e(t)=\max \left\{(1-t)^{\kappa}, 0\right\}$ shows (cf. $[10$, p. 42$])$.

Now there arises the question if one can improve (5) in the sense that the difference of the differentiation orders $\beta+1$ and $\kappa$ becomes small. It suggests itself to use the $W B V_{\beta, q}^{0}$-spaces (which essentially obey Sobolev embedding relations) and to conjecture that

$$
\begin{aligned}
M_{p}^{p}(\mathrm{rad}) \subset W B V_{\kappa, q}^{0}, \quad 1 \leqslant q<\infty, \kappa<n\left(\frac{1}{p}-\frac{1}{2}\right)+\left(\frac{1}{2}-\frac{1}{q^{\prime}}\right), \\
1<p<\frac{2 n}{n+1} .
\end{aligned}
$$

But (6) is not true for any $q\langle\infty, p$ near 1 . Namely, consider for $\alpha>-1$ the function $g_{\alpha}(y)=\max \left\{\left(1-|y|^{2}\right)^{\alpha}, 0\right\}$ with $g_{\alpha}^{\hat{\alpha}}(|z|)=C_{\alpha} K_{\alpha+n / 2}(|z|)$ (see [8, p. 171]). Since for large $t$, all $\kappa>0, g_{\alpha}{ }^{\wedge(k)}(t)=O\left(t^{-\alpha-(n+1) / 2}\right)$, it would follow by (6) that $\hat{g_{\alpha}} \notin M_{p}^{p}$ if $\alpha+(n+1) / 2<\kappa<n(1 / p-1 / 2)+\left(1 / 2-1 / q^{\prime}\right)$. But this is a contradiction if $p$ is near $1, q<\infty$ fixed, $\alpha$ near -1 , to $g_{\alpha}$ $\in\left[L^{1}\right]^{\wedge} \subset M_{p}^{p}$ for each $\alpha>-1$.

Since the right side of (5) cannot be improved in the above sense, let us try with the left side and conjecture that

$$
\begin{aligned}
W_{\beta, q}^{0} \subset M_{p}^{p}(\mathrm{rad}), \quad 1 \leqslant q \leqslant \infty, \beta>n\left(\frac{1}{p}-\frac{1}{2}\right)+ & \left(\frac{1}{2}-\frac{1}{q^{\prime}}\right), \\
& 1<p<\frac{2 n}{n+1} .
\end{aligned}
$$

This time (7) is not true for $q>2$. For consider the famous example

$$
f(|z|) \equiv \varphi(|z|)\left(1+|z|^{2}\right)^{-\gamma} e^{i|z|^{\alpha}}, \quad \alpha>0, \gamma>0,
$$


where $\varphi(|z|)$ is a smooth function which vanishes near the origin and is 1 for sufficiently large $|z|$. Observing $f^{(\beta)}(t)=O\left(t^{\beta(\alpha-1)-2 \gamma}\right)$ for large $t$, it would follow by (7) that $f \in M_{p}^{p}$ if $n(1 / p-1 / 2)+\left(1 / 2-1 / q^{\prime}\right)<\beta \leqslant 2 \gamma / \alpha$. In case $2 \gamma / \alpha n$ is near $(1 / p-1 / 2), q>2$ fixed, this is a contradiction to the result of S. Wainger (see [7, p. 113]) that $f \notin M_{p}^{p}$ if $1 / p-1 / 2>2 \gamma / \alpha n$. There are reasons to conjecture that (7) holds for $q=2$ (and hence also for $1 \leqslant q<2$ ); this would harmonize with various known Hörmander multiplier conditions; see, e.g., [7, p. 96], [3]; also the Herz conjecture concerning the Bochner-Riesz kernel would follow in the prescribed $(\alpha, p)$-range, i.e., $\max \left\{\left(1-|z|^{2}\right)^{\alpha}, 0\right\}$ $\in M_{p}^{p}$ if $\alpha>n(1 / p-1 / 2)-1 / 2$ and otherwise $\notin M_{p}^{p}$; a concise review concerning how much is already proved of the Herz conjecture is contained in Fefferman [4]; observe also the recent progress of Tomas [9].

Concluding, let us briefly mention inclusion relations for radial $M_{p}^{q}$ multipliers, where we omit an analogous discussion:

$$
\begin{aligned}
\left\{e ;\left\|t^{n / q^{\prime}} e(t)\right\|_{\infty}+\int_{0}^{\infty} t^{\beta+n / q^{\prime}}\left|d e^{(\beta)}(t)\right|<\infty\right\} \subset M_{1}^{q}(\mathrm{rad}) \subset W B V_{\kappa, \infty}^{n / q^{\prime}}, \\
1 \leqslant q<\frac{2 n}{n+1}, \quad \beta>n\left(\frac{1}{q}-\frac{1}{2}\right)-\frac{1}{2}, \quad \kappa<n\left(\frac{1}{q}-\frac{1}{2}\right)-\frac{1}{2}
\end{aligned}
$$

(the left inclusion is proved in [10, p. 83] in case $q=1$ and otherwise in [2]). If $1<p<q<2 n /(n+1)$ there holds (see [11])

$$
W B V_{\beta+1}^{n / r^{\prime}} \subset M_{p}^{q} \subset W B V_{\kappa, \infty}^{n / r^{\prime}}, \quad 1 / q=1 / p+1 / r-1,
$$

where $\beta>n(1 / r-1 / 2)-1 / 2, \kappa<n(1 / q-1 / 2)-1 / 2$.

\section{REFERENCES}

1. A. Bonami and J.-L. Clerc, Sommes de Cesàro et multiplicateurs des développements en harmoniques sphériques, Trans. Amer. Math. Soc. 183 (1973), 223-263. MR 49 \#3461.

2. P. L. Butzer, R. J. Nessel and W. Trebels, On radial $M_{p}^{q}$ Fourier multipliers, Math. Struct. Computer Math. -Math. Modelling, Bulgarian Acad. Sci., Sofia, 1975, pp. 187-193.

3. W. Connett and A. Schwartz, The theory of ultraspherical multipliers (to appear).

4. C. Fefferman, A note on spherical summation multipliers, Israel J. Math. 15 (1973), 44-52. MR 47 \#9160.

5. I. J. Schoenberg, Metric spaces and completely monotone functions, Ann. of Math. (2) 39 (1938), 811-841.

6. A. L. Schwartz, The smoothness of Hankel transforms, J. Math. Anal. Appl. 28 (1969), 500-507. MR 40 \#3204.

7. E. M. Stein, Singular integrals and differentiability properties of functions, Princeton Univ. Press, Princeton, N. J., 1970. MR 44 \# 7280.

8. E. M. Stein and G. Weiss, Introduction to Fourier analysis on Euclidean spaces, Princeton Univ. Press, Princeton, N. J., 1971. MR 46 \# 4102.

9. P. A. Tomas, On radial Fourier multipliers, Thesis, Cornell Univ., 1974.

10. W. Trebels, Multipliers for $(C, \alpha)$-bounded Fourier expansions in Banach spaces and approximation theory, Lecture Notes in Math., vol. 329, Springer-Verlag, Berlin and New York, 1973.

11. - On Fourier $M_{p}^{q}$ multiplier criteria of Marcinkiewicz type, Studia Math. 58 (1976), $7-19$.

Fachbereich Mathematik, T. H. Darmstadt, D-61 Darmstadt, Federal Republic of GermanY 